
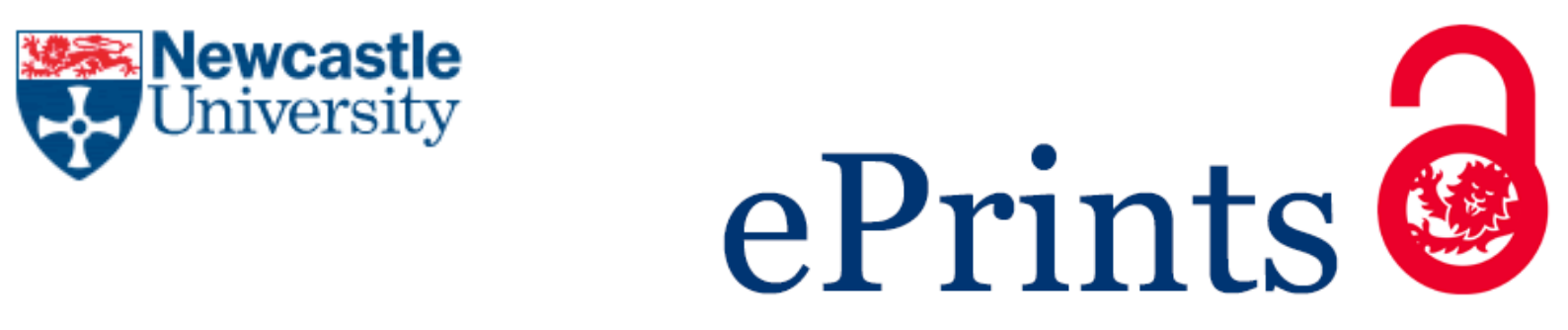

Ahmad Z, Zhang J, Kashiwao T, Bahadori A. Prediction of absorption and stripping factors in natural gas processing industries using feed forward artificial neural network. Petroleum Science and Technology 2016, 34(2), 105113.

\title{
Copyright:
}

This is an Accepted Manuscript of an article published by Taylor \& Francis in Petroleum Science and Technology on 22-02-16, available online:

http://www.tandfonline.com/10.1080/10916466.2015.1122628

Date deposited:

$05 / 04 / 2016$

Embargo release date:

22 February 2017

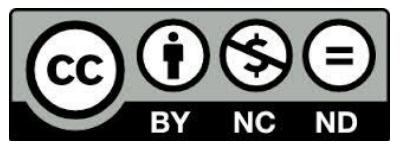

This work is licensed under a

Creative Commons Attribution-NonCommercial-NoDerivatives 4.0 International licence 


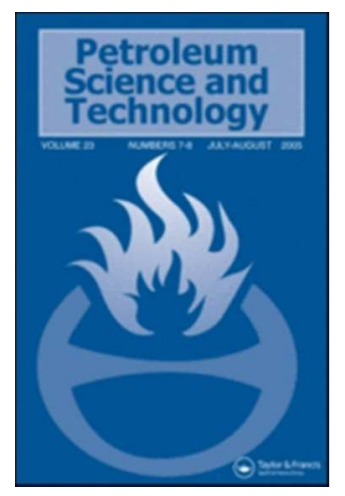

\section{Prediction of absorption and stripping factors in natural gas processing industries using feed forward artificial neural network}

\begin{tabular}{|r|l|}
\hline Journal: & Petroleum Science and Technology \\
\hline Manuscript ID & LPET-2015-0860 \\
\hline Danuscript Type: & Original Papers \\
\hline Complete List of Authors: & $\begin{array}{l}\text { Bahadori, Alireza; Southern Cross University, } \\
\text { Ahmad, Zainal; Universiti Sains Malaysia } \\
\text { Zhang, Jie; University of Newcastle } \\
\text { KASHIWAO, Tomoaki; National Institute of Technology, Niihama College, } \\
\text { Department of Electronics and Control Engineering }\end{array}$ \\
\hline Keywords: & $\begin{array}{l}\text { natural gas processing, absorption, stripping, modeling, artificial neural } \\
\text { network }\end{array}$ \\
\hline &
\end{tabular}




\title{
Prediction of absorption and stripping factors in natural gas processing industries using feed forward artificial neural network
}

\author{
Zainal Ahmad ${ }^{1}$ and Jie Zhang ${ }^{2}$, Tomoaki Kashiwao ${ }^{3}$, Alireza Bahadori** ${ }^{4}$ \\ ${ }^{1}$ School of Chemical Engineering, , Engineering Campus, Universiti Sains Malaysia ,Seri Ampangan, 14300, \\ Nibong Tebal, Penang, Malaysia \\ E-mail: chzahmad@usm.my \\ ${ }^{2}$ School of Chemical Engineering and Advanced Materials, University of Newcastle, Newcastle upon Tyne NE1 \\ 7RU, UK \\ E-mail: jie.zhang@newcastle.ac.uk \\ ${ }^{3}$ Department of Electronics and Control Engineering, National Institute of Technology, Niihama College, 7-1 \\ Yagumo-cho, Niihama, Ehime 792-8580, Japan \\ ${ }^{* * 4}$ School of Environment, Science and Engineering, Southern Cross University, Lismore, NSW, Australia \\ Email: Alireza.bahadori@scu.edu.au, Tel: +61 266269347
}

\begin{abstract}
In dynamic simulators, mathematical models are applied in order to study the timedependent behaviour of a system, meaning the system process units and the corresponding control units. Absorption and stripping are the unit operations which are widely used in the natural gas processing industries. Many attempts have been made to define an average absorption factor method to short-cut the time consuming rigorous calculation procedures. One of the options for this complex engineering modelling problem is artificial intelligent (AI) approach. Artificial neural networks (ANN) have been shown to be able to approximate any continuous non-linear functions and have been used to build data base empirical models for non-linear processes. In this study, feedforward neural networks (FANN) models were used to model the absorption efficiency. The mean square error (MSE), residue analysis and coefficient determination based on the observed and prediction output is chosen as the performance criteria of model. It was found that the developed feedforward neural networks (FANN) models provided satisfactory model with the MSE and coefficient determination of 0.0003 and 0.9998 for new unseen data from literature respectively.
\end{abstract}

Keywords: Artificial Intelligent (AI), Feedforward Neural Network (FANN), Absorption; Absorption efficiency, Absorption stages, stripping

\section{Introduction}

Absorption is one of the unit operations which is widely used in the natural gas processing industries [1]. In an absorption column, rich gas enters the bottom of the absorber and flows 
upward contacting the counter-current lean oil stream. The lean oil preferentially absorbs the heavier components from the gas and is then termed as "rich oil" [2].

The rich oil is sent to a stripper (or still) where the absorbed components are removed by heating and/or stripping with steam. The lean oil is recycled to the absorber to complete the process loop [2]. For a given gas, the fraction of each component in the gas that is absorbed by the oil is a function of the equilibrium phase relationship of the components and lean oil, the relative flow rates, and the contact stages $[2,3]$.

With currently available computing power, the process unit models in a dynamic simulator still need to be simplified in comparison to steady-state models for the purpose of real-time process optimisation and control [4]. The challenge is to model the complex phenomena associated with the absorber unit without losing important information.

Another challenge is the validation of a dynamic model, because relevant dynamic data from existing plants are not available. However, steady-state performance can be validated since there are more adequate data and advanced models available [4].

Dynamic modelling and simulation has been a very time-consuming and labour intensive activity, one requiring highly skilled systems engineers and computer applications specialists [5]. Therefore one of the solution in dynamics modelling and simulation for the above problem is by utilising the capability of artificial intelligence (AI).

One of the most popular AI methods is Artificial Neural Networks (ANN) specsically using feedforward artificial neural network (FANN). These kind of models are inspired from the neurological system of humans and used to mimic the human neurological system. After all, it shows a remarkable success in the modeling and prediction of highly nonlinear systems [6]. Artificial neural networks have been shown to be able to approximate any continuous nonlinear functions and have been used to build data base empirical models for non-linear processes [7]. Hence what is a neural network? According to [8].

A neural network is a massive parallel-distributed processor that has a natural capability for storing experiential knowledge and making it available for use. It resembles the brain in two respects knowledge is acquired by the networks through a learning process. Interneuron connection strengths known as synaptic weights are used to store the knowledge'

Furthermore, the main advantage of neural network based process models is that they are easy to build. This feature is particularly useful when modelling complicated processes where detailed mechanistic models are difficult to develop. The greatest strength of neural network is that it has the ability to learn the system from the historical data.

The growing interest in applying FANN are due to the computing system that growth rapidly which enable the behaviour of the complex system to be modelled and predicted accurately. Furthermore the characteristic of neural network models being able to learn from examples themselves rather than having to programme the complex system also contributed the application of the models. The architecture of FANN vary from multilayer perceptron as shown in Figure 1, to radial basis function $(\mathrm{RBF})$ and also recurrent neural networks models [9]. Most of the applications of FANN are concentrated on the modelling and control of chemical processes using multilayer perceptron networks. 
The common systems used in the chemical processes are distillation columns, absorption and reactor systems (continuous stirred tank reactor (CSTR), bioreactor, and neutralising reactor). These processes are usually very nonlinear and nonlinear models have to be developed. Currently, applications of FANN in process modeling and control are quite significant in industry especially in model based predictive control (MBPC) $[10,11]$ and this is due to the ability of neural networks in modeling nonlinear processes [12].

FANN has also been used to model complex systems in bioprocess, for example FANN are used as a biosensor to predict the glucose and ethanol in certain range of substrate and the accuracy of the estimation was quite good [13].

Further, [14] apply FANN with the extended Kalman filter in the training to predict the production of the penicillin in a batch process. It is shown that FANN predictions are quite good even in the real data. Therefore the FANN modelling approach is implemented in this paper with the aim of enhancing the model accuracy of the absorption efficiency model prediction.

This paper is organised as follows. Section 2 presents the case study or the process covering the absorber and stripper calculation. The concept of feedforward neural network (FANN) modelling is presented in Section 3. The result and discussion of the proposed modelling are presented in Section 4. Finally, the last section concludes this paper.

\section{Trays or packed Column Absorber 2.1 Absorber Calculations}

Absorber and stripper calculations, like fractionation column calculations, can be accomplished with tray-by-tray material and energy balance models [2]. However, hand calculations can be performed to estimate the absorption of components in a lean oil absorber. The stripping operation is essentially the reverse of absorption and can be handled in a similar fashion. Many attempts have been made to define an "average" absorption factor method to short-cut the time consuming rigorous calculation procedures [2].

The sole restriction of such a method is how well the average factor, as it is defined, will represent the absorption that actually occurs. One of the simplest definitions of an average absorption factor $A_{F}$ is by $[16,17]$, where the average absorption factor is defined as:

$$
A_{F}=\frac{L_{O}}{K_{a v g} V_{n+1}}
$$

Or

$$
L_{o}=A_{F} \times K_{a v g} \times V_{n+1}
$$

Using an average absorption factor, the extraction of any component from a rich gas can be described by: 


$$
\frac{Y_{n+1}-Y_{1}}{Y_{n+1}-Y_{0}}=\frac{A_{F}{ }^{n+1}-A_{F}}{A_{F}{ }^{n+1}-1}=E_{a}
$$

Where

Proposed predictive tool can also be used to determine the number of trays required for a given lean oil rate or to calculate recoveries with a given oil rate and number of trays. Most problems in absorber operation are centred on oil quality and rates. Proper stripping of the oil is necessary to minimize lean oil losses to the gas and to maximize absorption capacity.

\subsection{Stripper Calculations}

In a calculation sense, a stripper is simply an upside-down absorber wherein solute is stripped from liquid into the gas. For hand calculations, a stripping factor is defined [2]:

$$
S_{T}=\frac{K V}{L}
$$

Then:

$$
\frac{X_{m+1}-X_{1}}{X_{m+1}-X_{0}}=\frac{S_{T}^{m+1}-S_{T}}{S_{T}^{m+1}-1}=E_{s}
$$

The proposed predictive tool can be used to perform stripper calculations in a similar manner to absorber calculations. The present steady state absorber/stripper model can be applied to study operability in absorber plants during the course of steady operation. However, in a dynamic model, mathematical models are applied in order to study the time-dependent behavior of a system, meaning the system process units and the corresponding control units.

\section{Feed Forward Neural Network (FANN) Model Development}

In this case study, 1620 sampling instances were generated from the absorption process with the model inputs being the number of stripping stages (or number of absorption stages) and the absorption (or stripping) factor. The model output is the absorption (or stripping) efficiency. Modelling data are from the case study given in the previous section and additional testing of the model is carried out on the new unseen data from [17].

All the data was normalized to zero mean with unit standard deviation to cope with the different magnitudes in the input and output data. Then, the input data were divided randomly using Matlab ${ }^{\mathrm{TM}}$ command divideint into three sets of data which are $70 \%$ (1134 samples) for training, $15 \%$ (243 sample) for testing, and 15\% (243 sample) for unseen validation as show in Figures 2,3 and 4 respectively.

Then the individual networks were trained by the Levenberg-Marquardt optimisation algorithm with regularisation and "early stopping". All weights and biases were randomly initialised in the range from -0.1 to 0.1 . The individual networks are single hidden layer feed 
forward neural networks (FANN). Hidden layer neurons use the logarithmic sigmoid activation function whereas output layer neurons use the linear activation function.

The number of hidden neurons was determined using cross validation. The numbers of hidden nodes is increased from 1 to 20 and the MSE and $R^{2}$ value for the training and testing data are calculated for each nodes respectively.

Then, the MSE and $R^{2}$ is plotted against the number of nodes. Different number of hidden neurons will gives the different MSE and $R^{2}$ value in training and testing data. The network with the lowest mean squared errors (MSE) on the training and testing data was considered as having the best network topology for this prediction. In addition, in assessing the developed models, MSE on the unseen validation data is used as the performance criterion as well as the new unseen data from the literature.

For this case study, the FANN is developed based on the discrete time of the process as the prediction output at time $(t), y(t)$, is predicted based on the process input at time $t, u(t)$, as follows:

$$
\hat{y}(t)=f\left[u_{1}(t), u_{2}(t), \ldots, u_{m}(t)\right]
$$

where $u(t)$ is the process input at time $(t)$, where for this case study is striping stages and stripping factors, $\hat{y}(t)$ is the predicted process output at time $t$, which is the stripping efficiency while $m$ is the number of the model inputs respectively where for this case study number of inputs is 2 .

\section{Results and discussions}

It was found that using 8 hidden neurons gives the least MSE on the training and testing data as shown in Table 1. Based on this, the final selected neural network has 8 hidden neurons. Thus, the performance of the final selected model based on the unseen data will be evaluated based on this final structures as well.

The data for model building, 1134 samples, were randomly partitioned into training data (70\%), testing data (15\%), and unseen validation data (15\%). By using the LevenbergMarquardt optimization method, the training stopped after 100 iterations with the mean square error (MSE) value of 0.0009 and the coefficient determination, $R^{2}$ equal to 0.9992 . The trained network was simulated by feeding it with all of the $70 \%$ percent training data. Then, the model was tested using $30 \%$ data points which contains 243 data points. The testing prediction data was observed with the MSE value of 0.0006 and $R^{2}$ equal to 0.9994 respectively.

Figure 5 presents a plot of the true and predicted stripping efficiency values on the training and testing data. It can be seen from Figure 5 that all the predicted values are close to the 
corresponding actual values, which means that the network has learned the input-output mappings with a good degree of accuracy. The model has been validated using $15 \%$ from the total data as an unseen data which is not included in the model development process as shown in Figure 6. In addition to that, another literature data was also used as a new unseen data to further evaluate the generalisation capability of the developed model. Those 2 validation data sets will be used to determine whether the generalization capability of the model developed using training and testing data is acceptable for this case study.

Figure 6 shows the model predictions and actual outputs in the validation data for FANN. It can clearly be seen that the FANN model performs quite well. The model predictions are almost the same as the experiment data. This clearly shows that the FANN is able to capture the underlying relationship between the process input and output variables. The residue analysis also shows that the offset in each data point is very small and is close to zero.

In order to test further the performance of the model, statistical analysis was carried out in terms of the mean squared errors (MSE), coefficient of determination ( $R^{2}$ analysis), and residue and $p$-value analysis. The overall statistical analysis results of MSE and coefficient of determination are shown in Table 2. It clearly shows that the MSE is relatively small and the coefficient determination $\left(R^{2}\right)$ is close to 1 for the new unseen data. The p-values is very small, smaller than 0.05, indicating that the model and actual data are in $95 \%$ confident limit and this were supported by the residue analysis in Figure 6. It is shown that the FANN model is able to generalize on new unseen data and predict significantly well even though using real process data from literature.

\section{Conclusions}

A feedforward neural network (FANN) was developed to model the performance of an absorption process using experimental data, which was subjected to a series of different process operating conditions. The inputs to the network are the absorption or stripping stages and absorption or stripping factors, and the output is the absorption or stripping stage efficiency. The Levenberg-Marquardt optimization technique was used together with the 'early stopping' and regularisation methods to improve the robustness of the network. Application to the additional unseen data from the literature shows that feedforward neural networks (FANN) is able to capture and generalize the real process. The SSE is very small and the coefficient of determination is closed to 1 . These results are consistent with the pvalues and residue analysis. The presented study indicates that feedforward neural networks (FANN) can effectively model this process.

\section{Acknowledgement}

The authors would like to acknowledge the support received from the Universiti Sains Malaysia (USM) and Newcastle University, United Kingdom.

\section{Nomenclature:}


$A_{F}:$ Absorption factor

$E_{a}:$ Absorption efficiency

$E_{s}:$ Stripping efficiency

K: K-value

$L_{O}:$ liquid reflux rate, moles/unit time

$L$ :liquid rate, moles/unit time

$L_{m+1}$ :rich oil entering the stripper, moles/unit time

$\mathrm{m}$ : number of stripping stages

$\mathrm{n}$ : number of absorber stages

$\mathrm{N}_{m}$ : minimum number of theoretical stages

$\mathrm{R}$ : reflux ratio, moles of reflux divided by moles of net overhead product

$S$ : number of stages

$\mathrm{S}_{T}:$ stripping factor

$\mathrm{X}$ : liquid rate, moles/unit time

$\mathrm{X}_{m+1}$ : moles of a component in the rich oil entering a stripper per mole of rich oil entering the stripper

$\mathrm{X}_{1}$ : moles of a component in the lean oil per mole of rich oil

Xo : moles of a component in the liquid in equilibrium with the stripping medium per mole of entering rich oil

$\mathrm{Y}_{i}$ : moles of any component in the lean gas leaving the absorber per mole of rich gas

$\mathrm{Y}_{n+1}$ : moles of any component in the entering rich gas per mole of rich gas

Yo : moles of any component in the gas in equilibrium with the entering lean oil, per mole of rich gas

\section{Subscripts}

Avg: average

i : any component

$\mathrm{L}:$ liquid

$\mathrm{m}:$ number of stripping stages

$\mathrm{n}$ : number of absorber stages

\section{References}

Oyenekan B. A.,and Rochelle G. T. "Alternative stripper configurations for $\mathrm{CO}_{2}$ capture by aqueous amines", AIChE Journal 53 (12)(2007)pp. 3144 - 3154.

GPSA Engineering Databook, 2004

Bahadori A, Vuthaluru HB, Simple methodology for sizing of absorbers for TEG (triethylene glycol) gas dehydration systems, Energy 34 (2009a) 1910-1916. 
Kvamsdal, H.M. . Jakobsen J.P and Hoff, K.A. , Dynamic modeling and simulation of a $\mathrm{CO}_{2}$ absorber column for post-combustion $\mathrm{CO}_{2}$ capture, Chemical Engineering and Processing 48 (2009) 135-144.

Bahadori, a \& Vuthaluru, HB 2010, 'A new method for prediction of absorption/stripping factors', Computers \& Chemical Engineering, vol. 34, no. 10, pp 1731-1736.

Khuan, L. Y., Hamzah, N., \& Jailani, R. (2002). Prediction of water quality index (WQI) based on artificial neural network (ANN). In 2002 Student Conference on Research and Development Proceedings, Shah Alam, Malaysla (pp. 157-161). IEEE. doi:10.1109/SCORED.2002.1033081

Hertz J, Krogh A and Palmer R G., Introduction to the Theory of Neural Computation, Addison-Wesley, Redwood City, CA, 1991.

Haykin S. Neural Networks, Macmillan College Publication Company, 1994.

Hagan M T, Demuth H B and De Jesus O. 2002. An introduction to the use of neural networks in control system. International Journal of Robust and Nonlinear Control 12: 959-985.

Chen J and Yea Y. 2002. Neural network-based predictive control for multivariables processes. Chemical Engineering and Communication 189: 866-894.

Xiong Q and Jutan A. 2002. Grey-box modelling and control of chemical processes. Chemical Engineering Science 57: 1027-1039.

Shaw A M, Doyle F J and Schwaber J S. 1997. A dynamic neural networks approach to nonlinear process modelling. Computers and Chemical Engineering 21(4): 371-385.

Lobanov A V, Borisov I A, Gordon S H, Greene R V, Leathers T D and Reshetilov A N. 2001. Analysis of ethanol-glucose mixtures by two microbial sensors: applications of chemometrics and artificial neural networks for data processing. Biosensor and Bioelectronics 16: 1001-1007.

Scheffer R and Filho M. 2001. Process identification of a fed-batch penicilin production process-training with the extended Kalman filter. Mujtaba I M and Hussain M A (Eds). In Application of Neural Networks and Other Learning Technologies in Process Engineering, Imperial College Press, London:99-118.

Hitsov, I, Maere, T and Desitter, K. 2015. Modelling approaches in membrane distillation: a critical review, Separation and Purification Technology, 142:48-64

Kremser, A., "Theoretical analysis of absorption process", National Petro. News, 22(21)(1930), 48,.

Brown, G. G., and Souders, M., "Fundamental design of absorbing and stripping columns for complex vapors", Ind. Eng. Chem. 24 (1932), 519. 


1
2
3
4
5
6
7
8
9
10
11
12
13
14
15
16
17
18
19
20
21
22
23
24
25
26
27
28
29
30
31
32
33
34
35
36
37
38
39
40
41
42
43
44
45
46
47
48
49
50
51
52
53
54
55
56
57
58
60

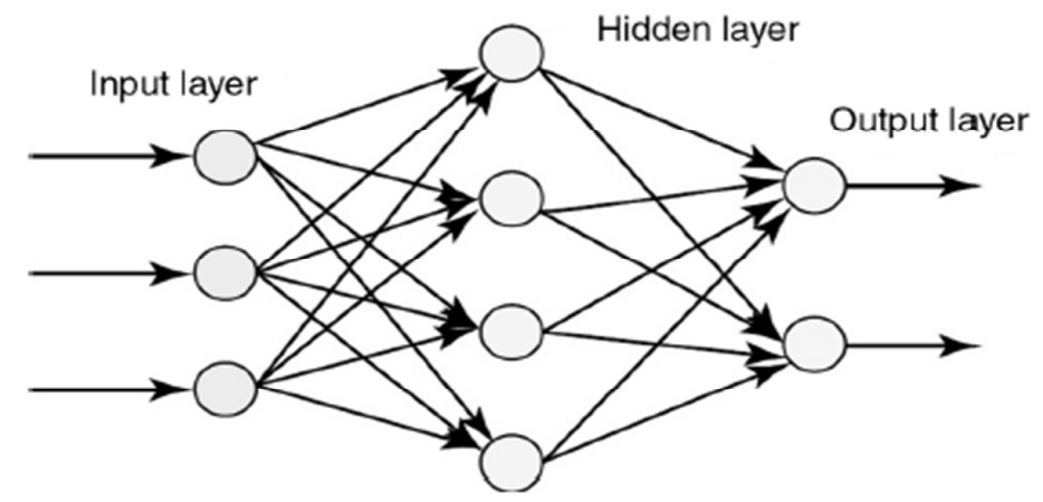

Figure 1: Multilayer artificial neural network with a single hidden layer [15] 

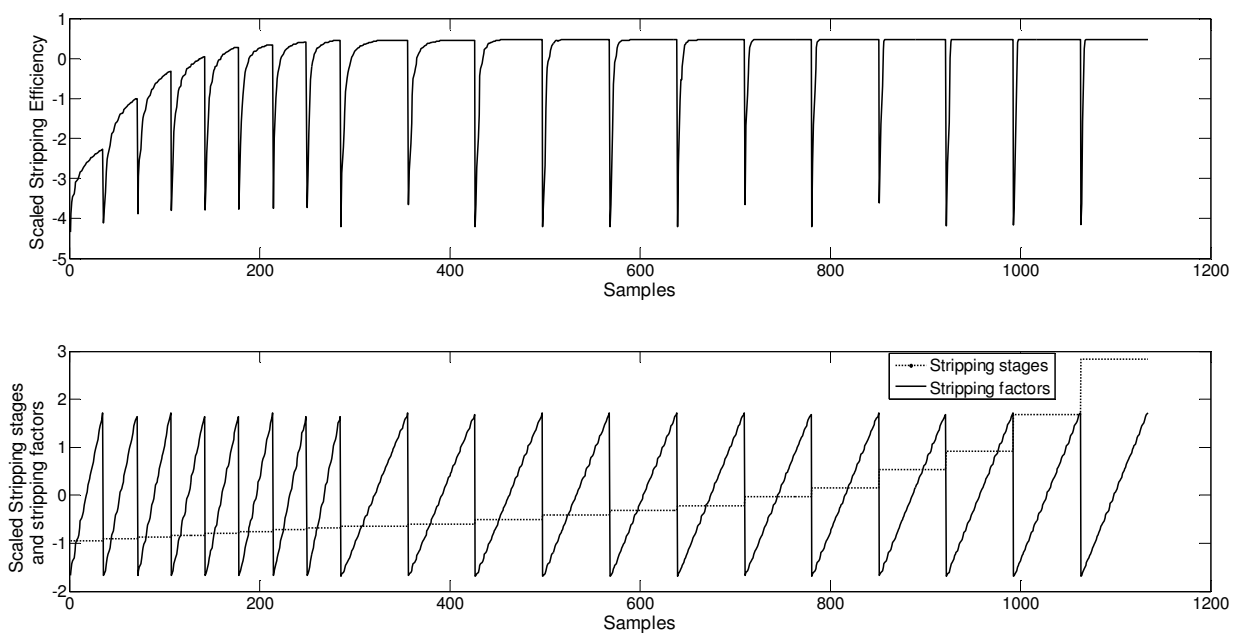

Figure 2: Scaled input and output training data 

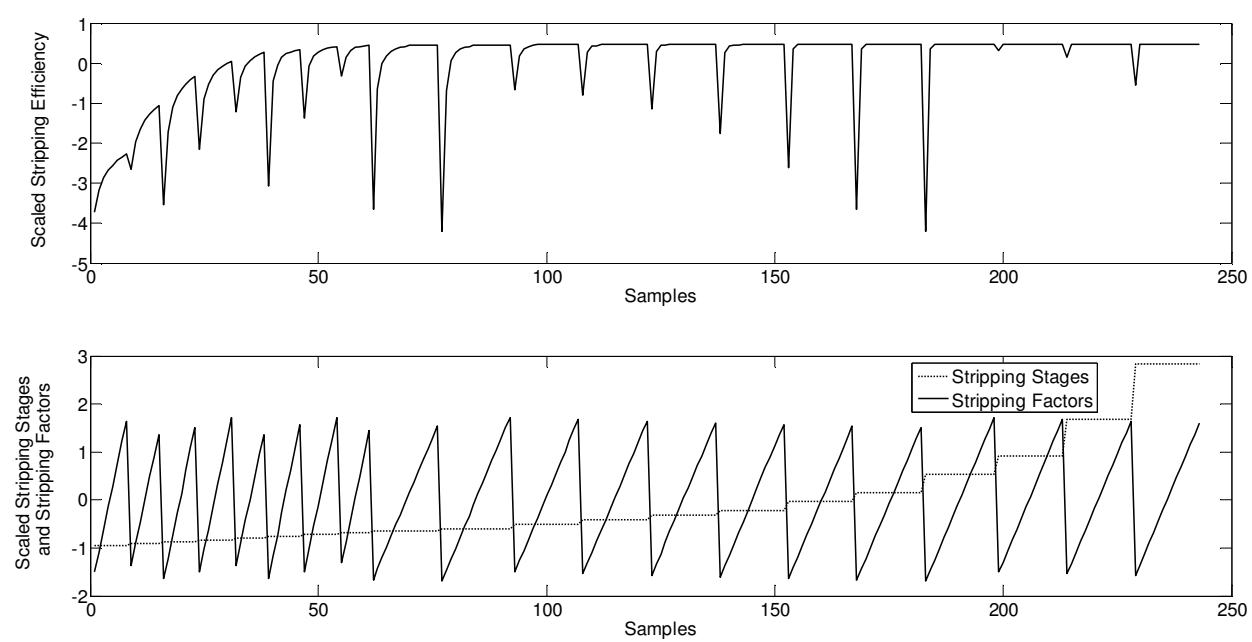

Figure 3: Scaled input and output for testing data 
Figure 4: Scaled input and output for validation data 

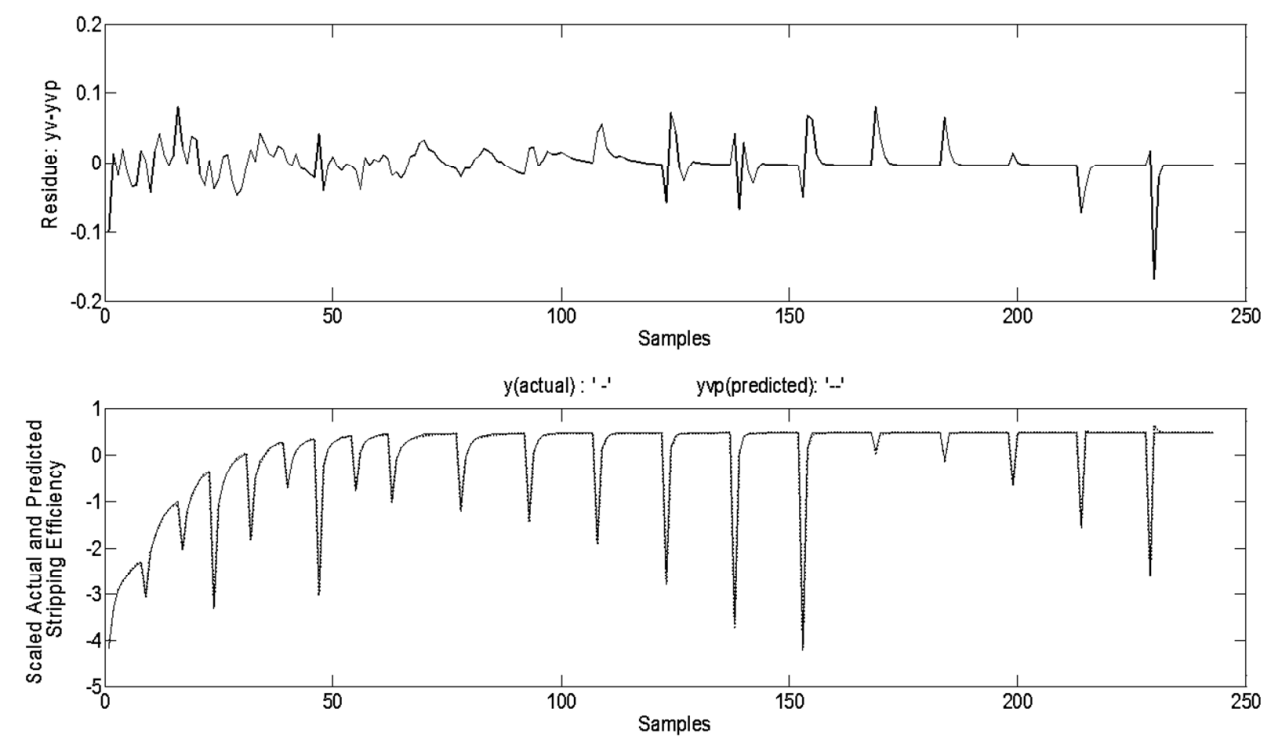

Figure 5: Actual and predicted for training and testing data 


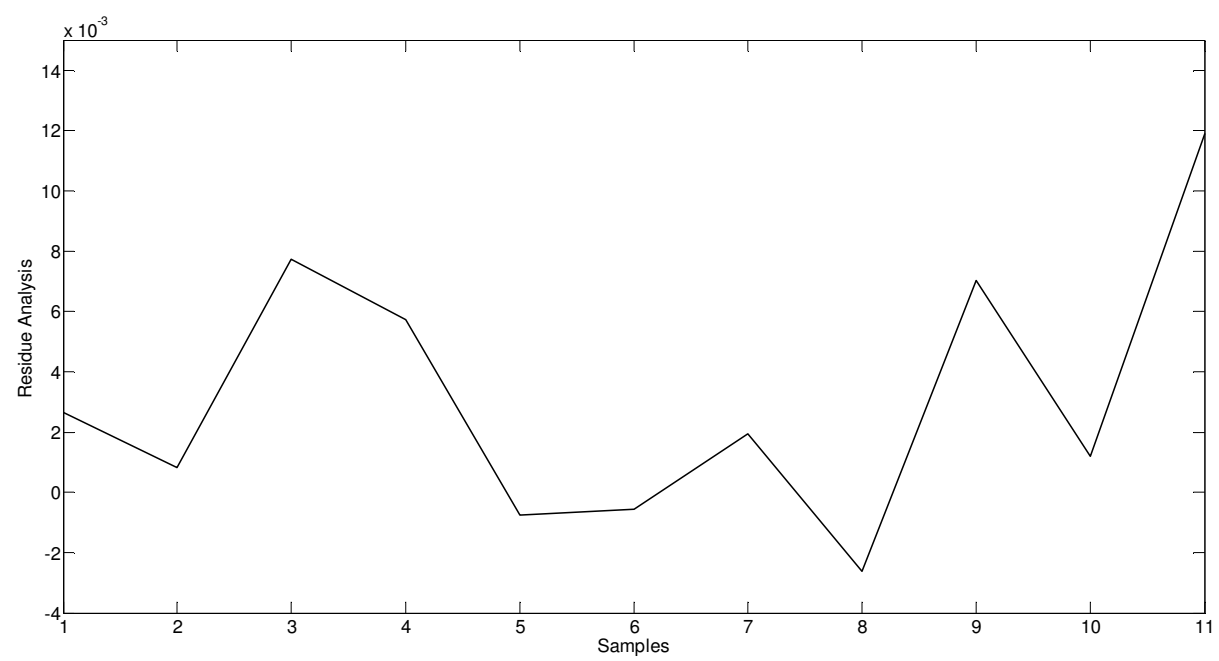

Figure 6: Actual and predicted for validation data

1
2
3
4
5
6
7
8
9
10
11
12
13
14
15
16
17
18
19
20
21
22
23
24
25
26
27
28
29
30
31
32
33
34
35
36
37
38
39
40
41
42
43
44
45
46
47
48
49
50
51
52
53
54
55
56
57
58
60


Table 1: MSE for neural networks with different numbers of hidden neurons

\begin{tabular}{|c|c|c|c|}
\hline Hidden nodes & MSE (Training) & MSE (Testing) & MSE (Train+Test) \\
\hline 1 & 0.0007 & 0.0002 & 0.0009 \\
\hline 2 & 0.0014 & 0.0012 & 0.0026 \\
\hline 3 & 0.0009 & 0.0007 & 0.0016 \\
\hline 4 & 0.0006 & 0.0003 & 0.0009 \\
\hline 5 & 0.0015 & 0.0013 & 0.0028 \\
\hline 6 & 0.0023 & 0.0022 & 0.0046 \\
\hline 7 & 0.0007 & 0.0004 & 0.0011 \\
\hline 8 & 0.0005 & 0.0003 & 0.0008 \\
\hline 9 & 0.0006 & 0.0003 & 0.0010 \\
\hline 10 & 0.0005 & 0.0002 & 0.0008 \\
\hline 11 & 0.0006 & 0.0004 & 0.0010 \\
\hline 12 & 0.0091 & 0.0062 & 0.0152 \\
\hline 13 & 0.0009 & 0.0007 & 0.0016 \\
\hline 14 & 0.0011 & 0.0009 & 0.0020 \\
\hline 15 & 0.0100 & 0.0059 & 0.0159 \\
\hline 16 & 0.0092 & 0.0064 & 0.0156 \\
\hline 17 & 0.0178 & 0.0145 & 0.0323 \\
\hline 18 & 0.0179 & 0.0147 & 0.0325 \\
\hline 19 & 0.5023 & 0.4315 & 0.9338 \\
\hline 20 & 0.5080 & 0.4352 & 0.9433 \\
\hline
\end{tabular}

URL: http://mc.manuscriptcentral.com/Ipet Email: jamessp8@aol.com 
Table 2: FANN model performance on the new unseen data and Statistical analysis for new unseen data $[2,16$, and 17]

\begin{tabular}{|c|c|c|c|c|}
\hline $\begin{array}{c}\text { Number of } \\
\text { absorption/stripping } \\
\text { stages }\end{array}$ & $\begin{array}{l}\text { Absorption or } \\
\text { stripping factor }\end{array}$ & $\begin{array}{c}\text { Reported absorption } \\
\text { or stripping } \\
\text { efficiency (fraction) } \\
{[2,16,17]}\end{array}$ & $\begin{array}{c}\text { FANN } \\
\text { predicted } \\
\text { values }\end{array}$ & $\begin{array}{c}\text { Absolute } \\
\text { deviation } \\
\text { percentage }\end{array}$ \\
\hline 0.4 & 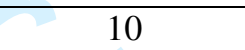 & 0.631503793 & 0.628851496 & 0.420 \\
\hline 0.6 & 20 & 0.845671811 & 0.844852747 & 0.097 \\
\hline 0.8 & 1.2 & 0.487758561 & 0.480022454 & 1.586 \\
\hline 1 & 6 & 0.868347362 & 0.862613684 & 0.660 \\
\hline 1.2 & 10 & 0.946202581 & 0.946956599 & 0.080 \\
\hline 1.4 & 16.8 & 0.983512804 & 0.984065285 & 0.056 \\
\hline 1.6 & 19.6 & 0.994966419 & 0.993026541 & 0.195 \\
\hline 2 & 14 & 0.993515174 & 0.99612215 & 0.262 \\
\hline 2.5 & 1.2 & 0.77627358 & 0.769239411 & 0.906 \\
\hline 5 & 0.8 & 0.732181329 & 0.730986621 & 0.163 \\
\hline 10 & 1 & 0.909949504 & 0.898050404 & 1.308 \\
\hline \multicolumn{4}{|c|}{ Average Absolute Deviation percentage (AADP) } & 0.521 \\
\hline \multicolumn{3}{|c|}{\begin{tabular}{|l|l} 
Details & \\
\end{tabular}} & \multicolumn{2}{|l|}{ Values } \\
\hline \multicolumn{2}{|c|}{$R^{2}$} & & 0.9999 & \\
\hline \multicolumn{2}{|c|}{ MSE } & & 0.0003 & \\
\hline \multicolumn{2}{|c|}{$p$-value } & & \multicolumn{2}{|l|}{$7.5257 \times 10^{-16}$} \\
\hline
\end{tabular}

URL: http://mc.manuscriptcentral.com/Ipet Email: jamessp8@aol.com 